\title{
ON SOME OSTROWSKI TYPE INEQUALITIES VIA MONTGOMERY IDENTITY AND TAYLOR'S FORMULA II
}

\author{
A. AGLIĆ ALJINOVIĆ, J. PEČARIĆ AND A.VUKELIĆ
}

\begin{abstract}
A new extension of the weighted Montgomery identity is given, by using Taylor's formula and used to obtain some Ostrowski type inequalities and estimations of the difference of two integral means.
\end{abstract}

\section{Introduction}

Let $f:[a, b] \rightarrow \mathbb{R}$ be differentiable on $[a, b]$, and $f^{\prime}:[a, b] \rightarrow \mathbb{R}$ integrable on $[a, b]$. Then the Montgomery identity holds [7]

$$
f(x)=\frac{1}{b-a} \int_{a}^{b} f(t) d t+\int_{a}^{b} P(x, t) f^{\prime}(t) d t
$$

where $P(x, t)$ is the Peano kernel, defined by

$$
P(x, t)= \begin{cases}\frac{t-a}{b-a}, & a \leq t \leq x, \\ \frac{t-b}{b-a}, & x<t \leq b .\end{cases}
$$

Now, let's suppose $w:[a, b] \rightarrow[0, \infty\rangle$ is some probability density function, i.e. integrable function satisfying $\int_{a}^{b} w(t) d t=1$, and $W(t)=\int_{a}^{t} w(x) d x$ for $t \in[a, b], W(t)=0$ for $t<a$ and $W(t)=1$ for $t>b$. The following identity (given by Pečarić in [8]) is the weighted generalization of Montgomery identity

$$
f(x)=\int_{a}^{b} w(t) f(t) d t+\int_{a}^{b} P_{w}(x, t) f^{\prime}(t) d t
$$

where the weighted Peano kernel is

$$
P_{w}(x, t)= \begin{cases}W(t), & a \leq t \leq x, \\ W(t)-1, & x<t \leq b,\end{cases}
$$

Received and revised February 10, 2004.

2000 Mathematics Subject Classification. 26D15, 26D20

Key words and phrases. Taylor's formula, weighted Montgomery identity. 
In this paper we will give an extension of weighted Montgomery identity using the Taylor's formula with respect to $a$ and $b$ (Section 2.) and further, obtain some new Ostrowski type inequalities (Section 3.), as well as some generalizations of the estimations of the difference of two weighted integral means (Section 4.). In paper [2] the same was done using an extension of weighted Montgomery identity via Taylor's formula with respect to $x$.

\section{An extension of Montgomery identity via Taylor's formula}

Theorem 1. Let $f: I \rightarrow \mathbb{R}$ be such that $f^{(n-1)}$ is absolutely continuous for some $n \geq 2, I \subset \mathbb{R}$ an open interval, $a, b \in I, a<b, w:[a, b] \rightarrow[0, \infty\rangle$ is some probability density function. Then the following identity holds

$$
\begin{aligned}
f(x)= & \int_{a}^{b} w(t) f(t) d t+\sum_{i=0}^{n-2} \frac{f^{(i+1)}(a)}{(i+1) !} \int_{a}^{x} w(s)\left((x-a)^{i+1}-(s-a)^{i+1}\right) d s \\
& +\sum_{i=0}^{n-2} \frac{f^{(i+1)}(b)}{(i+1) !} \int_{x}^{b} w(s)\left((x-b)^{i+1}-(s-b)^{i+1}\right) d s \\
& +\frac{1}{(n-1) !} \int_{a}^{b} T_{w, n}(x, s) f^{(n)}(s) d s
\end{aligned}
$$

where

$$
T_{w, n}(x, s)= \begin{cases}\int_{x}^{s} w(u)(u-s)^{n-1} d u+W(x)(x-s)^{n-1}, & a \leq s \leq x, \\ \int_{x}^{s} w(u)(u-s)^{n-1} d u+(W(x)-1)(x-s)^{n-1}, & x<s \leq b .\end{cases}
$$

Proof. If we apply Taylor's formula with $f^{\prime}(t)$, and replace $n$ with $n-1(n \geq 2)$ we have

$$
\begin{aligned}
f^{\prime}(t) & =\sum_{i=0}^{n-2} \frac{f^{(i+1)}(a)}{i !}(t-a)^{i}+\int_{a}^{t} f^{(n)}(s) \frac{(t-s)^{n-2}}{(n-2) !} d s \\
& =\sum_{i=0}^{n-2} \frac{f^{(i+1)}(b)}{i !}(t-b)^{i}-\int_{t}^{b} f^{(n)}(s) \frac{(t-s)^{n-2}}{(n-2) !} d s .
\end{aligned}
$$


By putting these two formulae in the weighted Montgomery identity (1.1) we obtain

$$
\begin{aligned}
f(x)= & \int_{a}^{b} w(t) f(t) d t \\
& +\sum_{i=0}^{n-2} \frac{f^{(i+1)}(a)}{i !} \int_{a}^{x}(t-a)^{i} W(t) d t+\sum_{i=0}^{n-2} \frac{f^{(i+1)}(b)}{i !} \int_{x}^{b}(t-b)^{i}(W(t)-1) d t \\
& +\int_{a}^{x} W(t)\left(\int_{a}^{t} f^{(n)}(s) \frac{(t-s)^{n-2}}{(n-2) !} d s\right) d t \\
& -\int_{x}^{b}(W(t)-1)\left(\int_{t}^{b} f^{(n)}(s) \frac{(t-s)^{n-2}}{(n-2) !} d s\right) d t .
\end{aligned}
$$

Now,

$$
\begin{aligned}
\int_{a}^{x}(t-a)^{i} W(t) d t & =\int_{a}^{x}(t-a)^{i}\left(\int_{a}^{t} w(s) d s\right) d t=\int_{a}^{x} w(s)\left(\int_{s}^{x}(t-a)^{i} d t\right) d s \\
& =\frac{1}{i+1} \int_{a}^{x} w(s)\left((x-a)^{i+1}-(s-a)^{i+1}\right) d s
\end{aligned}
$$

and similar

$$
\int_{x}^{b}(t-b)^{i}(W(t)-1)=\frac{1}{i+1} \int_{x}^{b} w(s)\left((x-b)^{i+1}-(s-b)^{i+1}\right) d s .
$$

Further, we have

$$
\int_{a}^{x} W(t)\left(\int_{a}^{t} f^{(n)}(s)(t-s)^{n-2} d s\right) d t=\int_{a}^{x} f^{(n)}(s)\left(\int_{s}^{x} W(t)(t-s)^{n-2} d t\right) d s
$$

and

$$
\begin{aligned}
& \int_{s}^{x} W(t)(t-s)^{n-2} d t=\int_{s}^{x}\left(\int_{a}^{t} w(u) d u\right)(t-s)^{n-2} d t \\
= & \int_{a}^{s} w(u)\left(\int_{s}^{x}(t-s)^{n-2} d t\right) d u+\int_{s}^{x} w(u)\left(\int_{u}^{x}(t-s)^{n-2} d t\right) d u \\
= & \int_{a}^{s} w(u) \frac{(x-s)^{n-1}}{n-1} d u+\int_{s}^{x} w(u) \frac{(x-s)^{n-1}-(u-s)^{n-1}}{n-1} d u \\
= & \frac{(x-s)^{n-1}}{n-1} W(x)-\int_{s}^{x} w(u) \frac{(u-s)^{n-1}}{n-1} d u .
\end{aligned}
$$

Similar

$$
\begin{aligned}
& -\int_{x}^{b}(W(t)-1)\left(\int_{t}^{b} f^{(n)}(s)(t-s)^{n-2} d s\right) d t \\
= & \int_{x}^{b} f^{(n)}(s)\left(\int_{x}^{s}(1-W(t))(t-s)^{n-2} d t\right) d s
\end{aligned}
$$


and

$$
\int_{x}^{s}(1-W(t))(t-s)^{n-2} d t=(W(x)-1) \frac{(x-s)^{n-1}}{n-1}+\int_{x}^{s} w(u) \frac{(u-s)^{n-1}}{n-1} d u .
$$

So the reminder in the weighted Taylor formula is

$$
\begin{aligned}
& \frac{1}{(n-1) !}\left[\int_{a}^{b} f^{(n)}(s)\left(\int_{x}^{s} w(u)(u-s)^{n-1} d u\right) d s\right. \\
& \left.+W(x) \int_{a}^{x} f^{(n)}(s)(x-s)^{n-1} d s+(W(x)-1) \int_{x}^{b} f^{(n)}(s)(x-s)^{n-1} d s\right] .
\end{aligned}
$$

Remark 1. In the special case, if we take $w(t)=\frac{1}{b-a}, t \in[a, b]$ the identity $(2.1)$ reduces to

$$
\begin{aligned}
f(x)= & \frac{1}{b-a} \int_{a}^{b} f(t) d t+\sum_{i=0}^{n-2} \frac{f^{(i+1)}(a)}{i !(i+2)} \frac{(x-a)^{i+2}}{b-a}-\sum_{i=0}^{n-2} \frac{f^{(i+1)}(b)}{i !(i+2)} \frac{(x-b)^{i+2}}{b-a} \\
& +\frac{1}{(n-1) !} \int_{a}^{b} T_{n}(x, s) f^{(n)}(s) d s,
\end{aligned}
$$

where

$$
T_{n}(x, s)= \begin{cases}-\frac{(x-s)^{n}}{n(b-a)}+\frac{x-a}{b-a}(x-s)^{n-1}, & a \leq s \leq x, \\ -\frac{(x-s)^{n}}{n(b-a)}+\frac{x-b}{b-a}(x-s)^{n-1}, & x<s \leq b .\end{cases}
$$

\section{The Ostrowski Type Inequalities}

In this section we generalize the results from $[1],[5],[6]$.

Theorem 2. Suppose that all the assumptions of Theorem 1 hold. Additionally assume that $(p, q)$ is a pair of conjugate exponents, that is $1 \leq p, q \leq \infty, \frac{1}{p}+\frac{1}{q}=1$. Then we have

$$
\begin{aligned}
& \mid f(x)-\int_{a}^{b} w(t) f(t) d t-\sum_{i=0}^{n-2} \frac{f^{(i+1)}(a)}{(i+1) !} \int_{a}^{x} w(s)\left((x-a)^{i+1}-(s-a)^{i+1}\right) d s \\
& -\sum_{i=0}^{n-2} \frac{f^{(i+1)}(b)}{(i+1) !} \int_{x}^{b} w(s)\left((x-b)^{i+1}-(s-b)^{i+1}\right) d s \mid \\
& \leq \frac{1}{(n-1) !}\left(\int_{a}^{b}\left|T_{w, n}(x, s)\right|^{q} d s\right)^{\frac{1}{q}}\left\|f^{(n)}\right\|_{p} .
\end{aligned}
$$


The constant $\frac{1}{(n-1) !}\left(\int_{a}^{b}\left|T_{w, n}(x, s)\right|^{q} d s\right)^{\frac{1}{q}}$ is sharp for $1<p \leq \infty$ and the best possible for $p=1$.

Proof. We use the identity (2.1) and apply the Hölder inequality to obtain

$$
\begin{aligned}
& \mid f(x)-\int_{a}^{b} w(t) f(t) d t-\sum_{i=0}^{n-2} \frac{f^{(i+1)}(a)}{(i+1) !} \int_{a}^{x} w(s)\left((x-a)^{i+1}-(s-a)^{i+1}\right) d s \\
& -\sum_{i=0}^{n-2} \frac{f^{(i+1)}(b)}{(i+1) !} \int_{x}^{b} w(s)\left((x-b)^{i+1}-(s-b)^{i+1}\right) d s \mid \\
= & \left|\frac{1}{(n-1) !} \int_{a}^{b} T_{w, n}(x, s) f^{(n)}(s) d s\right| \leq \frac{\left\|f^{(n)}\right\|_{p}}{(n-1) !}\left(\int_{a}^{b}\left|T_{w, n}(x, s)\right|^{q} d s\right)^{\frac{1}{q}}
\end{aligned}
$$

Let's denote $C_{1}(s)=\frac{1}{(n-1) !} T_{w, n}(x, s)$. For the proof of the sharpness of the constant $\left(\int_{a}^{b}\left|C_{1}(s)\right|^{q} d s\right)^{\frac{1}{q}}$ we will find a function $f$ for which the equality in (3.1) is obtained.

For $1<p<\infty$ take $f$ to be such that

$$
f^{(n)}(s)=\operatorname{sgn} C_{1}(s) \cdot\left|C_{1}(s)\right|^{\frac{1}{p-1}} .
$$

For $p=\infty$ take

$$
f^{(n)}(s)=\operatorname{sgn} C_{1}(s)
$$

For $p=1$ we shall prove that

$$
\left|\int_{a}^{b} C_{1}(s) f^{(n)}(s) d s\right| \leq \max _{s \in[a, b]}\left|C_{1}(s)\right|\left(\int_{a}^{b}\left|f^{(n)}(s)\right| d s\right)
$$

is the best possible inequality. Suppose that $\left|C_{1}(s)\right|$ attains its maximum at $s_{0} \in[a, b]$. First we assume that $C_{1}\left(s_{0}\right)>0$. For $\varepsilon$ such that $0<\varepsilon<b-s_{0}$ define $f_{\varepsilon}(s)$ by

$$
f_{\varepsilon}(s)= \begin{cases}0, & a \leq s \leq s_{0} \\ \frac{1}{\varepsilon n !}\left(s-s_{0}\right)^{n}, & s_{0} \leq s \leq s_{0}+\varepsilon \\ \frac{1}{n !}\left(s-s_{0}\right)^{n-1}, & s_{0}+\varepsilon \leq s \leq b .\end{cases}
$$

Then, for $\varepsilon$ small enough

$$
\left|\int_{a}^{b} C_{1}(s) f^{(n)}(s) d s\right|=\left|\int_{s_{0}}^{s_{0}+\varepsilon} C_{1}(s) \frac{1}{\varepsilon} d s\right|=\frac{1}{\varepsilon} \int_{s_{0}}^{s_{0}+\varepsilon} C_{1}(s) d s .
$$

Now, from inequality (3.2) we have

$$
\frac{1}{\varepsilon} \int_{s_{0}}^{s_{0}+\varepsilon} C_{1}(s) d s \leq C_{1}\left(s_{0}\right) \int_{s_{0}}^{s_{0}+\varepsilon} \frac{1}{\varepsilon} d s=C_{1}\left(s_{0}\right) .
$$


Since,

$$
\lim _{\varepsilon \rightarrow 0} \frac{1}{\varepsilon} \int_{s_{0}}^{s_{0}+\varepsilon} C_{1}(s) d s=C_{1}\left(s_{0}\right)
$$

the statement follows. In case $C_{1}\left(s_{0}\right)<0$, we take

$$
f_{\varepsilon}(s)= \begin{cases}\frac{1}{n !}\left(s-s_{0}-\varepsilon\right)^{n-1}, & a \leq s \leq s_{0}, \\ -\frac{1}{\varepsilon n !}\left(s-s_{0}-\varepsilon\right)^{n}, & s_{0} \leq s \leq s_{0}+\varepsilon, \\ 0, & s_{0}+\varepsilon \leq s \leq b,\end{cases}
$$

and the rest of proof is the same as above.

The Beta and the incomplete Beta function of Euler type are defined by

$$
B(x, y)=\int_{0}^{1} t^{x-1}(1-t)^{y-1} d t, \quad B_{r}(x, y)=\int_{0}^{r} t^{x-1}(1-t)^{y-1} d t, \quad x, y>0
$$

and

is a real positive valued integral.

$$
\Psi_{r}(x, y)=\int_{0}^{r} t^{x-1}(1+t)^{y-1} d t
$$

Corollary 1. Let $f: I \rightarrow \mathbb{R}$ be such that $f^{(n-1)}$ is absolutely continuous for some $n \geq 2, I \subset \mathbb{R}$ an open interval, $a, b \in I, a<b$ and $(p, q)$ a pair of conjugate exponents, $1<p \leq \infty$. Then we have

$$
\begin{aligned}
& \left|f(x)-\frac{1}{b-a} \int_{a}^{b} f(t) d t-\sum_{i=0}^{n-2} \frac{f^{(i+1)}(a)}{i !(i+2)} \frac{(x-a)^{i+2}}{b-a}+\sum_{i=0}^{n-2} \frac{f^{(i+1)}(b)}{i !(i+2)} \frac{(x-b)^{i+2}}{b-a}\right| \\
\leq & \frac{1}{(b-a)(n-1) !}\left\{\left((x-a)^{q n+1}+(b-x)^{q n+1}\right) n^{q(n-1)+1}\right. \\
& \left.\left(B(q+1, q(n-1)+1)-B_{\frac{n-1}{n}}(q+1, q(n-1)+1)\right)\right\}^{\frac{1}{q}}\left\|f^{(n)}\right\|_{p}
\end{aligned}
$$

and the constant on the right hand side of the inequality is sharp. For $p=1$ we have

$$
\begin{aligned}
& \left|f(x)-\frac{1}{b-a} \int_{a}^{b} f(t) d t-\sum_{i=0}^{n-2} \frac{f^{(i+1)}(a)}{i !(i+2)} \frac{(x-a)^{i+2}}{b-a}+\sum_{i=0}^{n-2} \frac{f^{(i+1)}(b)}{i !(i+2)} \frac{(x-b)^{i+2}}{b-a}\right| \\
\leq & \frac{n-1}{(b-a) n !} \max \left\{(x-a)^{n},(b-x)^{n}\right\}\left\|f^{(n)}\right\|_{1}
\end{aligned}
$$

and the constant on the right hand side of the inequality is the best possible.

Proof. We apply the inequality (3.1) with $w(t)=\frac{1}{b-a}, t \in[a, b]$ and use (2.2)

$$
\begin{aligned}
\int_{a}^{b}\left|T_{w, n}(x, s)\right|^{q} d s= & \int_{a}^{x}\left|-\frac{(x-s)^{n}}{n(b-a)}+\frac{x-a}{b-a}(x-s)^{n-1}\right|^{q} d s \\
& +\int_{x}^{b}\left|-\frac{(x-s)^{n}}{n(b-a)}+\frac{x-b}{b-a}(x-s)^{n-1}\right|^{q} d s .
\end{aligned}
$$


First with substitution $(x-a)-(x-s)=n t(x-a)$ we have

$$
\begin{aligned}
& \int_{a}^{x}\left|-\frac{(x-s)^{n}}{n}+(x-a)(x-s)^{n-1}\right|^{q} d s \\
= & \int_{a}^{x}(x-s)^{q(n-1)}\left((x-a)-\frac{(x-s)}{n}\right)^{q} d s \\
= & (x-a)^{q n+1} n^{q(n-1)+1}\left(B(q+1, q(n-1)+1)-B_{\frac{n-1}{n}}(q+1, q(n-1)+1)\right)
\end{aligned}
$$

Similar with $n(b-x)-(s-x)=n t(b-x)$ we get

$$
\begin{aligned}
& \int_{x}^{b}\left|-\frac{(x-s)^{n}}{n(b-a)}+\frac{x-b}{b-a}(x-s)^{n-1}\right|^{q} d s \\
= & (b-x)^{q n+1} n^{q(n-1)+1}\left(B(q+1, q(n-1)+1)-B_{\frac{n-1}{n}}(q+1, q(n-1)+1)\right) .
\end{aligned}
$$

Finally

$$
\begin{aligned}
\int_{a}^{b}\left|T_{w, n}(x, s)\right|^{q} d s= & \frac{1}{(b-a)^{q}}\left[(x-a)^{q n+1}+(b-x)^{q n+1}\right] n^{q(n-1)+1} \\
& \cdot\left(B(q+1, q(n-1)+1)-B_{\frac{n-1}{n}}(q+1, q(n-1)+1)\right)
\end{aligned}
$$

and the first inequality follows from the Theorem 2 .

For $p=1$

$$
\begin{array}{r}
\sup _{s \in[a, b]}\left|T_{w, n}(x, s)\right|=\max \left\{\sup _{s \in[a, x]}\left|-\frac{(x-s)^{n}}{n(b-a)}+\frac{x-a}{b-a}(x-s)^{n-1}\right|,\right. \\
\left.\sup _{s \in[x, b]}\left|-\frac{(x-s)^{n}}{n(b-a)}+\frac{x-b}{b-a}(x-s)^{n-1}\right|\right\} .
\end{array}
$$

By an elementary calculation we get

$$
\begin{aligned}
& \sup _{s \in[a, x]}\left|-\frac{(x-s)^{n}}{n(b-a)}+\frac{x-a}{b-a}(x-s)^{n-1}\right|=\frac{(n-1)(x-a)^{n}}{n(b-a)}, \\
& \sup _{s \in[x, b]}\left|-\frac{(x-s)^{n}}{n(b-a)}+\frac{x-b}{b-a}(x-s)^{n-1}\right|=\frac{(n-1)(b-x)^{n}}{n(b-a)} .
\end{aligned}
$$

Now, the second inequality follows from the Theorem 2.

Remark 2. With $n=2$ in Corollary 1 we get

$$
\begin{aligned}
& \left|f(x)-\frac{1}{b-a} \int_{a}^{b} f(t) d t+\frac{f^{\prime}(b)(x-b)^{2}-f^{\prime}(a)(x-a)^{2}}{2(b-a)}\right| \\
\leq & \frac{1}{(b-a)}\left\{\left((x-a)^{2 q+1}+(b-x)^{2 q+1}\right) 2^{q}(B(q+1, q+1))\right\}^{\frac{1}{q}}\left\|f^{\prime \prime}\right\|_{p}
\end{aligned}
$$


and for $p=1$ we have

$$
\begin{aligned}
& \left|f(x)-\frac{1}{b-a} \int_{a}^{b} f(t) d t+\frac{f^{\prime}(b)(x-b)^{2}-f^{\prime}(a)(x-a)^{2}}{2(b-a)}\right| \\
& \leq \frac{1}{2(b-a)} \max \left\{(x-a)^{2},(b-x)^{2}\right\}\left\|f^{\prime \prime}\right\|_{1} .
\end{aligned}
$$

If in inequality (3.3) we put $q=1$ we get

$$
\begin{aligned}
& \left|f(x)-\frac{1}{b-a} \int_{a}^{b} f(t) d t+\frac{f^{\prime}(b)(x-b)^{2}-f^{\prime}(a)(x-a)^{2}}{2(b-a)}\right| \\
\leq & \frac{1}{3(b-a)}\left[(x-a)^{3}+(b-x)^{3}\right]\left\|f^{\prime \prime}\right\|_{\infty} .
\end{aligned}
$$

Remark 3. If we apply (3.1) with $x=\frac{a+b}{2}$ we get the generalized midpoint inequality

$$
\begin{aligned}
& \mid f\left(\frac{a+b}{2}\right)-\int_{a}^{b} w(t) f(t) d t \\
& -\sum_{i=0}^{n-2} \frac{\left(\frac{b-a}{2}\right)^{i+1}}{(i+1) !}\left(f^{(i+1)}(a) W\left(\frac{a+b}{2}\right)+(-1)^{i+1} f^{(i+1)}(b)\left(1-W\left(\frac{a+b}{2}\right)\right)\right) \\
& \quad+\sum_{i=0}^{n-2} \frac{1}{(i+1) !}\left(f^{(i+1)}(a) \int_{a}^{\frac{a+b}{2}} w(s)(s-a)^{i+1} d s+f^{(i+1)}(b) \int_{\frac{a+b}{2}}^{b} w(s)(s-b)^{i+1} d s\right) \mid \\
& \leq \frac{1}{(n-1) !}\left(\int_{a}^{b}\left|T_{w, n}\left(\frac{a+b}{2}, s\right)\right|^{q} d s\right)^{\frac{1}{q}}\left\|f^{(n)}\right\|_{p} .
\end{aligned}
$$

If we additionally assume that $w(t)$ is symmetric on $[a, b]$ i.e. $w(t)=w(b-a-t)$ for every $t \in[a, b]$ this inequality reduces to

$$
\begin{aligned}
& \mid f\left(\frac{a+b}{2}\right)-\int_{a}^{b} w(t) f(t) d t \\
& \quad+\sum_{i=0}^{n-2} \frac{f^{(i+1)}(a)+(-1)^{i+1} f^{(i+1)}(b)}{(i+1) !}\left(\int_{a}^{\frac{a+b}{2}} w(s)(s-a)^{i+1} d s-\frac{1}{2}\left(\frac{b-a}{2}\right)^{i+1}\right) \mid \\
& \leq \frac{1}{(n-1) !}\left(\int_{a}^{b}\left|T_{w, n}\left(\frac{a+b}{2}, s\right)\right|^{q} d s\right)^{\frac{1}{q}}\left\|f^{(n)}\right\|_{p}
\end{aligned}
$$

and

$$
T_{w, n}\left(\frac{a+b}{2}, s\right)=\int_{\frac{a+b}{2}}^{s} w(u)(u-s)^{n-1} d u+\frac{1}{2}\left(\frac{a+b}{2}-s\right)^{n-1} \operatorname{sgn}\left(\frac{a+b}{2}-s\right) .
$$


For $n=2$ and $w(t)=\frac{1}{b-a}$ we get

$$
\begin{aligned}
& \left|f\left(\frac{a+b}{2}\right)-\frac{1}{b-a} \int_{a}^{b} f(t) d t+\frac{b-a}{8}\left(f^{\prime}(b)-f^{\prime}(a)\right)\right| \\
& \leq \frac{(b-a)^{1+\frac{1}{q}}}{2}(B(q+1, q+1))^{\frac{1}{q}}\left\|f^{\prime \prime}\right\|_{p}
\end{aligned}
$$

and for $q=1$

$$
\left|f\left(\frac{a+b}{2}\right)-\frac{1}{b-a} \int_{a}^{b} f(t) d t+\frac{b-a}{8}\left(f^{\prime}(b)-f^{\prime}(a)\right)\right| \leq \frac{(b-a)^{2}}{12}\left\|f^{\prime \prime}\right\|_{\infty} .
$$

For the generalized trapezoid inequality we apply equality (2.1) first with $x=a$, then with $x=b$ then add them up and divide by 2 . After applying the Hölder inequality we get

$$
\begin{aligned}
& \mid \frac{f(a)+f(b)}{2}-\int_{a}^{b} w(t) f(t) d t-\sum_{i=0}^{n-2} \frac{f^{(i+1)}(a)}{2(i+1) !}\left((b-a)^{i+1}-\int_{a}^{b} w(s)(s-a)^{i+1} d s\right) \\
& -\sum_{i=0}^{n-2} \frac{f^{(i+1)}(b)}{2(i+1) !}\left((-1)^{i+1}(b-a)^{i+1}-\int_{a}^{b} w(s)(s-b)^{i+1} d s\right) \mid \\
\leq & \frac{1}{2(n-1) !}\left(\int_{a}^{b}\left|T_{w, n}(a, s)+T_{w, n}(b, s)\right|^{q} d s\right)^{\frac{1}{q}}\left\|f^{(n)}\right\|_{p}
\end{aligned}
$$

and

$$
T_{w, n}(a, s)+T_{w, n}(b, s)=\int_{a}^{b} \operatorname{sgn}(s-u) w(u)(u-s)^{n-1} d u+(b-s)^{n-1}-(a-s)^{n-1} .
$$

Again, if we additionally assume that $w(t)$ is symmetric on $[a, b]$ this inequality reduces to

$$
\begin{aligned}
& \mid \frac{f(a)+f(b)}{2}-\int_{a}^{b} w(t) f(t) d t-\sum_{i=0}^{n-2} \frac{1}{2(i+1) !}\left(f^{(i+1)}(a)+(-1)^{i+1} f^{(i+1)}(b)\right) \\
& \cdot\left((b-a)^{i+1}-\int_{a}^{b} w(s)(s-a)^{i+1} d s\right) \mid \\
\leq & \frac{1}{2(n-1) !}\left(\int_{a}^{b}\left|T_{w, n}(a, s)+T_{w, n}(b, s)\right|^{q} d s\right)^{\frac{1}{q}}\left\|f^{(n)}\right\|_{p} .
\end{aligned}
$$

For $n=2, w(t)=\frac{1}{b-a}$ and $q=1$ we get

$$
\left|\frac{f(a)+f(b)}{2}-\frac{1}{b-a} \int_{a}^{b} f(t) d t+\frac{b-a}{4}\left(f^{\prime}(b)-f^{\prime}(a)\right)\right| \leq \frac{(b-a)^{2}}{3}\left\|f^{\prime \prime}\right\|_{\infty} .
$$




\section{The Estimation of the Difference of the Two Weighted Integral Means}

In this section we generalize the results from [3], [4]. We denote

$$
\begin{aligned}
t_{w, n}^{[a, b]}(x) & =\sum_{i=0}^{n-2} \frac{f^{(i+1)}(a)}{(i+1) !} \int_{a}^{x} w(s)\left((x-a)^{i+1}-(s-a)^{i+1}\right) d s \\
& +\sum_{i=0}^{n-2} \frac{f^{(i+1)}(b)}{(i+1) !} \int_{x}^{b} w(s)\left((x-b)^{i+1}-(s-b)^{i+1}\right) d s
\end{aligned}
$$

and

$$
D(w, u ; x)=\int_{a}^{b} w(t) f(t) d t-\int_{c}^{d} u(t) f(t) d t+t_{w, n}^{[a, b]}(x)-t_{u, n}^{[c, d]}(x),
$$

for function $f:[a, b] \rightarrow \mathbb{R}$ such that $f^{(n-1)}$ is absolutely continuous function for some $n \geq 2$.

For the two intervals $[a, b]$ and $[c, d]$ we have four possible cases if $[a, b] \cap[c, d] \neq \emptyset$. The first case is $[c, d] \subseteq[a, b]$ and the second $[a, b] \cap[c, d]=[c, b]$. Other two possible cases we simply get by change $a \leftrightarrow c, b \leftrightarrow d$.

Theorem 3. Let $f:[a, b] \cup[c, d] \rightarrow \mathbb{R}$ be such that $f^{(n-1)}$ is an absolutely continuous function for some $n \geq 2, w:[a, b] \rightarrow[0, \infty\rangle$ and $u:[c, d] \rightarrow[0, \infty\rangle$ some probability density functions, $W(t)=\int_{a}^{t} w(x) d x$ for $t \in[a, b], W(t)=0$ for $t<a$ and $W(t)=1$ for $t>b, U(t)=\int_{c}^{t} u(x) d x$ for $t \in[c, d], U(t)=0$ for $t<c$ and $U(t)=1$ for $t>d$. Then if $[a, b] \cap[c, d] \neq \emptyset$ and $x \in[a, b] \cap[c, d]$, we have

$$
D(w, u ; x)=\int_{\min \{a, c\}}^{\max \{b, d\}} K_{n}(x, s) f^{(n)}(s) d s
$$

where in case $[c, d] \subseteq[a, b]$

$$
K_{n}(x, s)= \begin{cases}-\frac{1}{(n-1) !}\left(\int_{x}^{s} w(t)(t-s)^{n-1} d t+W(x)(x-s)^{n-1}\right), & s \in[a, c], \\ -\frac{1}{(n-1) !}\left(\int_{x}^{s}(w(t)-u(t))(t-s)^{n-1} d t\right. & s \in\langle c, d], \\ \left.+(W(x)-U(x))(x-s)^{n-1}\right), & s \in\langle d, b], \\ -\frac{1}{(n-1) !}\left(\int_{x}^{s} w(t)(t-s)^{n-1} d t+(W(x)-1)(x-s)^{n-1}\right),\end{cases}
$$

and in case $[a, b] \cap[c, d]=[c, b]$

$$
K_{n}(x, s)= \begin{cases}-\frac{1}{(n-1) !}\left(\int_{x}^{s} w(t)(t-s)^{n-1} d t+W(x)(x-s)^{n-1}\right), & s \in[a, c], \\ -\frac{1}{(n-1) !}\left(\int_{x}^{s}(w(t)-u(t))(t-s)^{n-1} d t\right. & s \in\langle c, b], \\ \left.+(W(x)-U(x))(x-s)^{n-1}\right), & s \in\langle b, d] . \\ \frac{1}{(n-1) !}\left(\int_{x}^{s} u(t)(t-s)^{n-1} d t+(U(x)-1)(x-s)^{n-1}\right),\end{cases}
$$


Proof. We subtract identities (2.1) for interval $[a, b]$ and $[c, d]$, to get the formula (4.1).

Theorem 4. Assume $(p, q)$ is a pair of conjugate exponents, that is $1 \leq p, q \leq \infty$, $\frac{1}{p}+\frac{1}{q}=1$. Let $\left|f^{(n)}\right|^{p}:[a, b] \cup[c, d] \rightarrow \mathbb{R}$ be an R-integrable function for some $n \geq 2$. Then we have

$$
|D(w, u ; x)| \leq\left(\int_{\min \{a, c\}}^{\max \{b, d\}}\left|K_{n}(x, s)\right|^{q} d s\right)^{\frac{1}{q}}\left\|f^{(n)}\right\|_{p}
$$

for every $x \in[a, b] \cap[c, d]$. The constant $\left(\int_{\min \{a, c\}}^{\max \{b, d\}}\left|K_{n}(x, s)\right|^{q} d s\right)^{\frac{1}{q}}$ is sharp for $1<p \leq$ $\infty$ and the best possible for $p=1$.

Proof. Use the identity (4.1) and apply the Hölder inequality to obtain

$$
\begin{aligned}
|D(w, u ; x)| & \leq \int_{\min \{a, c\}}^{\max \{b, d\}}\left|K_{n}(x, s)\right|\left|f^{(n)}(s)\right| d s \\
& \leq\left(\int_{\min \{a, c\}}^{\max \{b, d\}}\left|K_{n}(x, s)\right|^{q} d s\right)^{\frac{1}{q}}\left\|f^{(n)}\right\|_{p},
\end{aligned}
$$

which proves the inequality. The proof for sharpness and the best possibility are similar as in Theorem 2.

\subsection{Case $[c, d] \subseteq[a, b]$}

Here we denote

$$
t_{n}^{[a, b]}(x)=\sum_{i=0}^{n-2} \frac{f^{(i+1)}(a)}{i !(i+2)} \frac{(x-a)^{i+2}}{b-a}-\sum_{i=0}^{n-2} \frac{f^{(i+1)}(b)}{i !(i+2)} \frac{(x-b)^{i+2}}{b-a}
$$

and

$$
D\left(\frac{1}{b-a}, \frac{1}{d-c} ; x\right)=\frac{1}{b-a} \int_{a}^{b} f(t) d t-\frac{1}{d-c} \int_{c}^{d} f(t) d t+t_{n}^{[a, b]}(x)-t_{n}^{[c, d]}(x) .
$$

Corollary 2. Assume $(p, q)$ is a pair of conjugate exponents. Let $\left|f^{(n)}\right|^{p}:[a, b] \rightarrow \mathbb{R}$ be an $R$-integrable function for some $n \geq 2,[c, d] \subseteq[a, b], x \in[c, d]$ and $s_{0}=\frac{b c-a d}{c-a+b-d}$. 
Then for $1<p \leq \infty$ and $x+n\left(s_{0}-x\right) \notin[c, d]$ we have

$$
\begin{aligned}
& \left|D\left(\frac{1}{b-a}, \frac{1}{d-c} ; x\right)\right| \leq \frac{n^{n-1+\frac{1}{q}}}{(n-1) !(b-a)} \\
& \cdot\left[(x-a)^{n q+1}\left(B_{1-\frac{x-c}{n(x-a)}}(q+1,(n-1) q+1)-B_{1-\frac{1}{n}}(q+1,(n-1) q+1)\right)\right. \\
& +\frac{(c-a+b-d)^{q}}{(d-c)^{q}}\left|x-s_{o}\right|^{n q+1}(B(q+1,(n-1) q+1) \\
& \left.-B_{r_{1}}(q+1,(n-1) q+1)+\Psi_{r_{2}}((n-1) q+1, q+1)\right) \\
& \left.+(b-x)^{n q+1}\left(B_{1-\frac{d-x}{n(b-x)}}(q+1,(n-1) q+1)-B_{1-\frac{1}{n}}(q+1,(n-1) q+1)\right)\right]^{\frac{1}{q}}\left\|f^{(n)}\right\|_{p}
\end{aligned}
$$

where for $x+n\left(s_{0}-x\right)<c, r_{1}=1-\frac{x-c}{n\left(x-s_{0}\right)}, r_{2}=\frac{d-x}{n\left(x-s_{0}\right)}$, while for $x+n\left(s_{0}-x\right)>$ $d, r_{1}=1-\frac{d-x}{n\left(s_{0}-x\right)}, r_{2}=\frac{x-c}{n\left(s_{0}-x\right)}$.

If $x+n\left(s_{0}-x\right) \in[c, d]$ we have

$$
\begin{aligned}
& \left|D\left(\frac{1}{b-a}, \frac{1}{d-c} ; x\right)\right| \leq \frac{n^{n-1+\frac{1}{q}}}{(n-1) !(b-a)} \\
& \cdot\left[(x-a)^{n q+1}\left(B_{1-\frac{x-c}{n(x-a)}}(q+1,(n-1) q+1)-B_{1-\frac{1}{n}}(q+1,(n-1) q+1)\right)\right. \\
& +\frac{(c-a+b-d)^{q}}{(d-c)^{q}}\left|x-s_{o}\right|^{n q+1}\left(\Psi_{r_{3}}((n-1) q+1, q+1)\right. \\
& \left.+B(q+1,(n-1) q+1)+\Psi_{r_{4}}(q+1,(n-1) q+1)\right) \\
& \left.+(b-x)^{n q+1}\left(B_{1-\frac{d-x}{n(b-x)}}(q+1,(n-1) q+1)-B_{1-\frac{1}{n}}(q+1,(n-1) q+1)\right)\right]^{\frac{1}{q}}\left\|f^{(n)}\right\|_{p}
\end{aligned}
$$

where for $x<s_{0}, r_{3}=\frac{x-c}{n\left(s_{0}-x\right)}, r_{4}=\frac{d-x}{n\left(s_{0}-x\right)}-1$, while for $s_{0}<x, r_{3}=\frac{d-x}{n\left(x-s_{0}\right)}, r_{4}=$ $\frac{x-c}{n\left(x-s_{0}\right)}-1$.

For $p=1$ we have

$$
\begin{aligned}
& \left|D\left(\frac{1}{b-a}, \frac{1}{d-c} ; x\right)\right| \leq \frac{1}{(b-a) n !} \max \left\{(n-1)(x-a)^{n},(n-1)(b-x)^{n},\right. \\
& \frac{c-a+b-d}{d-c}(x-c)^{n-1}\left|x-c+n\left(s_{0}-x\right)\right|, \frac{c-a+b-d}{d-c}\left|s_{0}-x\right|^{n}(n-1)^{n-1}, \\
& \left.\frac{c-a+b-d}{d-c}(d-x)^{n-1}\left|d-x+n\left(x-s_{0}\right)\right|\right\}\left\|f^{(n)}\right\|_{1} .
\end{aligned}
$$

Proof. We put $w(t)=\frac{1}{b-a}, t \in[a, b]$, and $u(t)=\frac{1}{d-c}, t \in[c, d]$ in the Theorem 4 . 
Thus we have $t_{n}^{[a, b]}(x)$ and $t_{n}^{[c, d]}(x)$ instead of $t_{w, n}^{[a, b]}(x)$ and $t_{u, n}^{[c, d]}(x)$ and

$$
\begin{aligned}
& \left(\int_{\min \{a, c\}}^{\max \{b, d\}}\left|K_{n}(x, s)\right|^{q} d s\right)^{\frac{1}{q}}=\frac{1}{(n-1) !}\left(\int_{a}^{c}\left|-\frac{(x-s)^{n}}{n(b-a)}+\frac{x-a}{b-a}(x-s)^{n-1}\right|^{q} d s\right. \\
& +\int_{c}^{d}\left|-\frac{(x-s)^{n}}{n(b-a)}+\frac{(x-s)^{n}}{n(d-c)}+\left(\frac{x-a}{b-a}-\frac{x-c}{d-c}\right)(x-s)^{n-1}\right|^{q} d s \\
& \left.+\int_{d}^{b}\left|-\frac{(x-s)^{n}}{n(b-a)}+\frac{x-b}{b-a}(x-s)^{n-1}\right|^{q} d s\right)^{\frac{1}{q}} .
\end{aligned}
$$

For the first integral let $x-a-\frac{x-s}{n}=t(x-a)$ so

$$
\begin{aligned}
I_{1} & =\int_{a}^{c}\left|-\frac{(x-s)^{n}}{n(b-a)}+\frac{x-a}{b-a}(x-s)^{n-1}\right|^{q} d s \\
& =\frac{(x-a)^{n q+1} n^{(n-1) q+1}}{(b-a)^{q}}\left(B_{1-\frac{x-c}{n(x-a)}}(q+1,(n-1) q+1)-B_{1-\frac{1}{n}}(q+1,(n-1) q+1)\right) .
\end{aligned}
$$

For the third integral let $b-x-\frac{s-x}{n}=t(b-x)$ and similar obtain

$$
\begin{aligned}
I_{3} & =\int_{d}^{b}\left|-\frac{(x-s)^{n}}{n(b-a)}+\frac{x-b}{b-a}(x-s)^{n-1}\right|^{q} d s \\
& =\frac{(b-x)^{n q+1} n^{(n-1) q+1}}{(b-a)^{q}}\left(B_{1-\frac{d-x}{n(b-x)}}(q+1,(n-1) q+1)-B_{1-\frac{1}{n}}(q+1,(n-1) q+1)\right) .
\end{aligned}
$$

As is $c-a+b-d>0$, the second integral is

$$
\begin{aligned}
I_{2} & =\int_{c}^{d}\left|-\frac{(x-s)^{n}}{n(b-a)}+\frac{(x-s)^{n}}{n(d-c)}+\left(\frac{x-a}{b-a}-\frac{x-c}{d-c}\right)(x-s)^{n-1}\right|^{q} d s \\
& =\frac{(c-a+b-d)^{q}}{n^{q}(b-a)^{q}(d-c)^{q}} \int_{c}^{d}\left|(x-s)^{n-1}\left(x-s+n\left(s_{0}-x\right)\right)\right|^{q} d s .
\end{aligned}
$$

Since $s_{0}-c=\frac{(d-c)(c-a)}{c-a+b-d} \geq 0$ and $d-s_{0}=\frac{(d-c)(b-d)}{c-a+b-d} \geq 0$, then $s_{0} \in[c, d]$. So we have four possible cases:

1. If $x+n\left(s_{0}-x\right)<c$, (then also $\left.x>s_{0}\right)$ we have

$$
\begin{aligned}
& \int_{c}^{d}\left|(x-s)^{n-1}\left(x-s+n\left(s_{0}-x\right)\right)\right|^{q} d s \\
= & \int_{c}^{x}(x-s)^{(n-1) q}\left(s-x+n\left(x-s_{0}\right)\right)^{q} d s+\int_{x}^{d}(s-x)^{(n-1) q}\left(s-x+n\left(x-s_{0}\right)\right)^{q} d s .
\end{aligned}
$$

Now, using the substitution $s-x+n\left(x-s_{0}\right)=n t\left(x-s_{0}\right)$ we get

$$
\begin{aligned}
& \int_{c}^{x}(x-s)^{(n-1) q}\left(s-x+n\left(x-s_{0}\right)\right)^{q} d s \\
= & \left(x-s_{0}\right)^{q n+1} n^{q n+1}\left(B(q+1,(n-1) q+1)-B_{1-\frac{x-c}{n\left(x-s_{0}\right)}}(q+1,(n-1) q+1)\right) .
\end{aligned}
$$


Similar, using the substitution $s-x=n t\left(x-s_{0}\right)$ we get

$$
\begin{aligned}
& \int_{x}^{d}(s-x)^{(n-1) q}\left(s-x+n\left(x-s_{0}\right)\right)^{q} d s \\
= & \left(x-s_{0}\right)^{q n+1} n^{q n+1} \Psi_{\frac{d-x}{n\left(x-s_{0}\right)}}((n-1) q+1, q+1) .
\end{aligned}
$$

2. If $x+n\left(s_{0}-x\right)>d$, (then also $\left.s_{0}>x\right)$ we have

$$
\begin{aligned}
& \int_{c}^{d}\left|(x-s)^{n-1}\left(x-s+n\left(s_{0}-x\right)\right)\right|^{q} d s \\
= & \int_{c}^{x}(x-s)^{(n-1) q}\left(x-s+n\left(s_{0}-x\right)\right)^{q} d s+\int_{x}^{d}(s-x)^{(n-1) q}\left(x-s+n\left(s_{0}-x\right)\right)^{q} d s .
\end{aligned}
$$

Using the substitution $x-s=n t\left(s_{0}-x\right)$ similar we get

$$
\begin{aligned}
& \int_{c}^{x}(x-s)^{(n-1) q}\left(x-s+n\left(s_{0}-x\right)\right)^{q} d s \\
= & \left(s_{0}-x\right)^{q n+1} n^{q n+1} \Psi \frac{x-c}{n\left(s_{0}-x\right)}((n-1) q+1, q+1)
\end{aligned}
$$

and using the substitution $x-s+n\left(s_{0}-x\right)=n t\left(s_{0}-x\right)$

$$
\begin{aligned}
& \int_{x}^{d}(s-x)^{(n-1) q}\left(x-s+n\left(s_{0}-x\right)\right)^{q} d s \\
= & \left(s_{0}-x\right)^{q n+1} n^{q n+1}\left(B(q+1,(n-1) q+1)-B_{1-\frac{d-x}{n\left(s_{0}-x\right)}}(q+1,(n-1) q+1)\right) .
\end{aligned}
$$

3. If $c \leq x+n\left(s_{0}-x\right) \leq d$ and $x \leq x+n\left(s_{0}-x\right)$, (so $\left.x<s_{0}\right)$ then

$$
\begin{aligned}
& \int_{c}^{d}\left|(x-s)^{n-1}\left(x-s+n\left(s_{0}-x\right)\right)\right|^{q} d s \\
= & \int_{c}^{x}(x-s)^{(n-1) q}\left(x-s+n\left(s_{0}-x\right)\right)^{q} d s \\
& +\int_{x}^{x+n\left(s_{0}-x\right)}(s-x)^{(n-1) q}\left(x-s+n\left(s_{0}-x\right)\right)^{q} d s \\
& +\int_{x+n\left(s_{0}-x\right)}^{d}(s-x)^{(n-1) q}\left(s-x+n\left(x-s_{0}\right)\right)^{q} d s .
\end{aligned}
$$

We already had the first integral (see (4.5)), for second let $x-s+n\left(s_{0}-x\right)=n t\left(s_{0}-x\right)$ and then

$$
\begin{aligned}
& \int_{x}^{x+n\left(s_{0}-x\right)}(s-x)^{(n-1) q}\left(x-s+n\left(s_{0}-x\right)\right)^{q} d s \\
= & \left(s_{0}-x\right)^{q n+1} n^{q n+1} B(q+1,(n-1) q+1),
\end{aligned}
$$


and for third let $s-x+n\left(x-s_{0}\right)=\operatorname{tn}\left(s_{0}-x\right)$ so

$$
\begin{aligned}
& \int_{x+n\left(s_{0}-x\right)}^{d}(s-x)^{(n-1) q}\left(s-x+n\left(x-s_{0}\right)\right)^{q} d s \\
= & \left(s_{0}-x\right)^{q n+1} n^{q n+1} \Psi_{\frac{d-x}{n\left(s_{0}-x\right)}-1}(q+1,(n-1) q+1) .
\end{aligned}
$$

4. If $c \leq x+n\left(s_{0}-x\right) \leq d$ and $x+n\left(s_{0}-x\right)<x$, (so $\left.s_{0}<x\right)$ then

$$
\begin{aligned}
& \int_{c}^{d}\left|(x-s)^{n-1}\left(x-s+n\left(s_{0}-x\right)\right)\right|^{q} d s \\
= & \int_{c}^{x+n\left(s_{0}-x\right)}(x-s)^{(n-1) q}\left(x-s+n\left(s_{0}-x\right)\right)^{q} d s \\
& +\int_{x+n\left(s_{0}-x\right)}^{x}(x-s)^{(n-1) q}\left(s-x+n\left(x-s_{0}\right)\right)^{q} d s \\
& +\int_{x}^{d}(s-x)^{(n-1) q}\left(s-x+n\left(x-s_{0}\right)\right)^{q} d s .
\end{aligned}
$$

Now, let $x-s+n\left(s_{0}-x\right)=\operatorname{tn}\left(x-s_{0}\right)$ so

$$
\begin{aligned}
& \int_{c}^{x+n\left(s_{0}-x\right)}(x-s)^{(n-1) q}\left(x-s+n\left(s_{0}-x\right)\right)^{q} d s \\
= & \left(x-s_{0}\right)^{q n+1} n^{q n+1} \Psi_{\frac{x-c}{n\left(x-s_{0}\right)}-1}(q+1,(n-1) q+1),
\end{aligned}
$$

and with $s-x+n\left(x-s_{0}\right)=n t\left(x-s_{0}\right)$ we get

$$
\begin{aligned}
& \int_{x+n\left(s_{0}-x\right)}^{x}(x-s)^{(n-1) q}\left(s-x+n\left(x-s_{0}\right)\right)^{q} d s \\
= & \left(x-s_{0}\right)^{q n+1} n^{q n+1} B(q+1,(n-1) q+1),
\end{aligned}
$$

and we already had the third integral before (4.4). Finally, by summing $I_{1}, I_{2}$, and $I_{3}$, the statement for $1<p \leq \infty$ follows. For $p=1$, we have

$$
\begin{aligned}
& \left\|K_{n}(x, s)\right\|_{\infty}=\sup _{s \in[a, b]}\left|K_{n}(x, s)\right| \\
& =\max \left\{\max _{s \in[a, c]}\left|-\frac{(x-s)^{n}}{n(b-a)}+\frac{x-a}{b-a}(x-s)^{n-1}\right|,\right. \\
& \max _{s \in[c, d]}\left|-\frac{(x-s)^{n}}{n(b-a)}+\frac{(x-s)^{n}}{n(d-c)}+\left(\frac{x-a}{b-a}-\frac{x-c}{d-c}\right)(x-s)^{n-1}\right|, \\
& \left.\max _{s \in[d, b]}\left|-\frac{(x-s)^{n}}{n(b-a)}+\frac{x-b}{b-a}(x-s)^{n-1}\right|\right\} \text {. }
\end{aligned}
$$

By an elementary calculation we get

$$
\max _{s \in[a, c]}\left|-\frac{(x-s)^{n}}{n(b-a)}+\frac{x-a}{b-a}(x-s)^{n-1}\right|=\frac{(n-1)(x-a)^{n}}{n(b-a)},
$$




$$
\begin{aligned}
& \max _{s \in[d, b]}\left|-\frac{(x-s)^{n}}{n(b-a)}+\frac{x-b}{b-a}(x-s)^{n-1}\right|=\frac{(n-1)(b-x)^{n}}{n(b-a)}, \\
& \max _{s \in[c, d]}\left|-\frac{(x-s)^{n}}{n(b-a)}+\frac{(x-s)^{n}}{n(d-c)}+\left(\frac{x-a}{b-a}-\frac{x-c}{d-c}\right)(x-s)^{n-1}\right| \\
= & \frac{c-a+b-d}{n(b-a)(d-c)} \max _{s \in[c, d]}\left|(x-s)^{n-1}\left(x-s+n\left(s_{0}-x\right)\right)\right| .
\end{aligned}
$$

Again, for calculating $\max _{s \in[c, d]}\left|(x-s)^{n-1}\left(x-s+n\left(s_{0}-x\right)\right)\right|$ we have four possible cases:

1. If $x+n\left(s_{0}-x\right)<c$, (then also $\left.x>s_{0}\right)$ we have

$$
\max _{s \in[c, x]}(x-s)^{n-1}\left(s-x+n\left(x-s_{0}\right)\right)=(x-M)^{n-1}\left(M-x+n\left(x-s_{0}\right)\right),
$$

where $M=\max \left\{c, x+(n-1)\left(s_{0}-x\right)\right\}$

$$
\max _{s \in[x, d]}(s-x)^{n-1}\left(s-x+n\left(x-s_{0}\right)\right)=(d-x)^{n-1}\left(d-x+n\left(x-s_{0}\right)\right) .
$$

2. If $x+n\left(s_{0}-x\right)>d$, (then also $\left.s_{0}>x\right)$

$$
\begin{gathered}
\max _{s \in[c, x]}(x-s)^{n-1}\left(x-s+n\left(s_{0}-x\right)\right)=(x-c)^{n-1}\left(x-c+n\left(s_{0}-x\right)\right), \\
\max _{s \in[x, d]}(s-x)^{n-1}\left(x-s+n\left(s_{0}-x\right)\right)=(m-x)^{n-1}\left(x-m+n\left(s_{0}-x\right)\right) .
\end{gathered}
$$

where $m=\min \left\{b, x+(n-1)\left(s_{0}-x\right)\right\}$

3. If $c \leq x+n\left(s_{0}-x\right) \leq d$ and $x \leq x+n\left(s_{0}-x\right)$, so $x<s_{0}$ then

$$
\begin{gathered}
\max _{s \in[c, x]}(x-s)^{n-1}\left(x-s+n\left(s_{0}-x\right)\right)=(x-c)^{n-1}\left(x-c+n\left(s_{0}-x\right)\right), \\
\max _{s \in\left[x, x+n\left(s_{0}-x\right)\right]}(s-x)^{n-1}\left(x-s+n\left(s_{0}-x\right)\right)=\left(s_{0}-x\right)^{n}(n-1)^{n-1}, \\
\max _{s \in\left[x+n\left(s_{0}-x\right), d\right]}(s-x)^{n-1}\left(s-x+n\left(x-s_{0}\right)\right)=(d-x)^{n-1}\left(d-x+n\left(x-s_{0}\right)\right) .
\end{gathered}
$$

4. If $c \leq x+n\left(s_{0}-x\right) \leq d$ and $x+n\left(s_{0}-x\right)<x$, so $s_{0}<x$ then

$$
\begin{gathered}
\max _{s \in\left[c, x+n\left(s_{0}-x\right)\right]}(x-s)^{n-1}\left(x-s+n\left(s_{0}-x\right)\right)=(x-c)^{n-1}\left(x-c+n\left(s_{0}-x\right)\right), \\
\max _{s \in\left[x+n\left(s_{0}-x\right), x\right]}(x-s)^{n-1}\left(s-x+n\left(x-s_{0}\right)\right)=\left(x-s_{0}\right)^{n}(n-1)^{n-1}, \\
\max _{s \in[x, d]}(s-x)^{n-1}\left(s-x+n\left(x-s_{0}\right)\right)=(d-x)^{n-1}\left(d-x+n\left(x-s_{0}\right)\right) .
\end{gathered}
$$

Thus, the proof is done. 
Remark 4. If we put $c=d=x$ as a limit case, the inequalities from Corollary 2 reduce to Ostrowski type inequalities from Corollary 1.

\subsection{Case $[a, b] \cap[c, d]=[c, b]$}

Corollary 3. Assume $(p, q)$ is a pair of conjugate exponents. Let $\left|f^{(n)}\right|^{p}:[a, d] \rightarrow \mathbb{R}$ be an $R$-integrable function for some $n \geq 2,[a, b] \cap[c, d]=[c, b], x \in[c, b]$. If $c-a+b-d=$ 0 then for $1<p \leq \infty$

$$
\begin{aligned}
& \left|D\left(\frac{1}{b-a}, \frac{1}{d-c} ; x\right)\right| \leq \frac{1}{(n-1) !} \\
& \cdot\left[\frac{(x-a)^{q n+1} n^{(n-1) q+1}}{(b-a)^{q}}\left(B_{1-\frac{x-c}{n(x-a)}}(q+1,(n-1) q+1)-B_{1-\frac{1}{n}}(q+1,(n-1) q+1)\right)\right. \\
& +\frac{c-a}{b-a}\left(\frac{(x-c)^{(n-1) q+1}+(b-x)^{(n-1) q+1}}{(n-1) q+1}\right) \\
& \left.+\frac{(d-x)^{n q+1} n^{(n-1) q+1}}{(d-c)^{q}}\left(B_{1-\frac{b-x}{n(d-x)}}(q+1,(n-1) q+1)-B_{1-\frac{1}{n}}(q+1,(n-1) q+1)\right)\right]^{\frac{1}{q}}\left\|f^{(n)}\right\|_{p}
\end{aligned}
$$

and if $c-a+b-d \neq 0$, let $s_{0}=\frac{b c-a d}{c-a+b-d}$. Then for $1<p \leq \infty$ and $x+n\left(s_{0}-x\right) \in[c, b]$ we have

$$
\begin{aligned}
& \left|D\left(\frac{1}{b-a}, \frac{1}{d-c} ; x\right)\right| \leq \frac{n^{n-1+\frac{1}{q}}}{(n-1) !} \\
& \cdot\left[\frac{(x-a)^{q n+1}}{(b-a)^{q}}\left(B_{1-\frac{x-c}{n(x-a)}}(q+1,(n-1) q+1)-B_{1-\frac{1}{n}}(q+1,(n-1) q+1)\right)\right. \\
& +\frac{|c-a+b-d|^{q}}{(b-a)^{q}(d-c)^{q}}\left|x-s_{o}\right|^{n q+1}\left(\Psi_{r_{1}}(q+1,(n-1) q+1)\right. \\
& \left.+B(q+1,(n-1) q+1)+\Psi_{r_{2}}((n-1) q+1, q+1)\right) \\
& \left.+\frac{(d-x)^{n q+1}}{(d-c)^{q}}\left(B_{1-\frac{b-x}{n(d-x)}}(q+1,(n-1) q+1)-B_{1-\frac{1}{n}}(q+1,(n-1) q+1)\right)\right]^{\frac{1}{q}}\left\|f^{(n)}\right\|_{p}
\end{aligned}
$$

where for $s_{0}>b, r_{1}=\frac{b-x}{n\left(s_{0}-x\right)}-1, r_{2}=\frac{x-c}{n\left(s_{0}-x\right)}$, while for $s_{0}<c, r_{1}=\frac{x-c}{n\left(x-s_{o}\right)}-1, r_{2}=$ $\frac{b-x}{n\left(x-s_{0}\right)}$. 
If $x+n\left(s_{0}-x\right) \notin[c, b]$ we have

$$
\begin{aligned}
& \left|D\left(\frac{1}{b-a}, \frac{1}{d-c} ; x\right)\right| \leq \frac{n^{n-1+\frac{1}{q}}}{(n-1) !} \\
& \cdot\left[\frac{(x-a)^{q n+1}}{(b-a)^{q}}\left(B_{1-\frac{x-c}{n(x-a)}}(q+1,(n-1) q+1)-B_{1-\frac{1}{n}}(q+1,(n-1) q+1)\right)\right. \\
& +\frac{|c-a+b-d|^{q}}{(b-a)^{q}(d-c)^{q}}\left|x-s_{o}\right|^{n q+1}\left(\Psi_{r_{3}}((n-1) q+1, q+1)\right. \\
& \left.+B(q+1,(n-1) q+1)-B_{r_{4}}(q+1,(n-1) q+1)\right) \\
& \left.+\frac{(d-x)^{n q+1}}{(d-c)^{q}}\left(B_{1-\frac{b-x}{n(d-x)}}(q+1,(n-1) q+1)-B_{1-\frac{1}{n}}(q+1,(n-1) q+1)\right)\right]^{\frac{1}{q}}\left\|f^{(n)}\right\|_{p}
\end{aligned}
$$

where for $x+n\left(s_{0}-x\right)>b, r_{3}=\frac{x-c}{n\left(s_{o}-x\right)}, r_{4}=1-\frac{b-x}{n\left(s_{0}-x\right)}$, while for $x+n\left(s_{0}-x\right)<$ $c, r_{3}=\frac{b-x}{n\left(x-s_{0}\right)}, r_{4}=1-\frac{x-c}{n\left(x-s_{0}\right)}$.

For $p=1$ and $c-a+b-d=0$ we have

$$
\begin{aligned}
& \left|D\left(\frac{1}{b-a}, \frac{1}{d-c} ; x\right)\right| \leq \frac{1}{n !} \max \left\{(n-1) \frac{(x-a)^{n}}{(b-a)},(n-1) \frac{(d-x)^{n}}{(d-c)},\right. \\
& \left.\frac{c-a}{b-a}(x-c)^{n-1}, \frac{c-a}{b-a}(b-x)^{n-1}\right\}\left\|f^{(n)}\right\|_{1}
\end{aligned}
$$

and for $p=1$ and $c-a+b-d \neq 0$

$$
\begin{aligned}
& \left|D\left(\frac{1}{b-a}, \frac{1}{d-c} ; x\right)\right| \\
\leq & \frac{1}{n !} \max \left\{(n-1) \frac{(x-a)^{n}}{(b-a)},(n-1) \frac{(d-x)^{n}}{(d-c)}, \frac{|c-a+b-d|}{(b-a)(d-c)}(x-c)^{n-1}\left|x-c+n\left(s_{0}-x\right)\right|,\right. \\
& \left.\frac{|c-a+b-d|}{(b-a)(d-c)}\left|s_{0}-x\right|^{n}(n-1)^{n-1}, \frac{|c-a+b-d|}{(b-a)(d-c)}(d-x)^{n-1}\left|d-x+n\left(x-s_{0}\right)\right|\right\}\left\|f^{(n)}\right\|_{1} .
\end{aligned}
$$

Proof. We put $w(t)=\frac{1}{b-a}, t \in[a, b]$, and $u(t)=\frac{1}{d-c}, t \in[c, d]$ in the Theorem 4 . Thus

$$
\begin{aligned}
& \left(\int_{\min \{a, c\}}^{\max \{b, d\}}\left|K_{n}(x, s)\right|^{q} d s\right)^{\frac{1}{q}}=\frac{1}{(n-1) !}\left(\int_{a}^{c}\left|-\frac{(x-s)^{n}}{n(b-a)}+\frac{x-a}{b-a}(x-s)^{n-1}\right|^{q} d s\right. \\
& +\int_{c}^{b}\left|-\frac{(x-s)^{n}}{n(b-a)}+\frac{(x-s)^{n}}{n(d-c)}+\left(\frac{x-a}{b-a}-\frac{x-c}{d-c}\right)(x-s)^{n-1}\right|^{q} d s \\
& \left.+\int_{b}^{d}\left|\frac{(x-s)^{n}}{n(d-c)}-\frac{x-d}{d-c}(x-s)^{n-1}\right|^{q} d s\right)^{\frac{1}{q}} .
\end{aligned}
$$


$I_{1}$ is the same as in the proof of the Corollary 2 and with substitution $d-x-\frac{s-x}{n}=$ $t(d-x)$

$$
\begin{aligned}
I_{3} & =\int_{b}^{d}\left|\frac{(x-s)^{n}}{n(d-c)}-\frac{x-d}{d-c}(x-s)^{n-1}\right|^{q} d s \\
& =\frac{(d-x)^{n q+1} n^{(n-1) q+1}}{(d-c)^{q}}\left(B_{1-\frac{b-x}{n(d-x)}}(q+1,(n-1) q+1)-B_{1-\frac{1}{n}}(q+1,(n-1) q+1)\right) .
\end{aligned}
$$

If $c-a+b-d=0$ the second integral is

$$
\begin{aligned}
I_{2} & =\int_{c}^{b}\left|-\frac{(x-s)^{n}}{n(b-a)}+\frac{(x-s)^{n}}{n(d-c)}+\left(\frac{x-a}{b-a}-\frac{x-c}{d-c}\right)(x-s)^{n-1}\right|^{q} d s \\
& =\frac{c-a}{b-a}\left(\frac{(x-c)^{(n-1) q+1}+(b-x)^{(n-1) q+1}}{(n-1) q+1}\right) .
\end{aligned}
$$

If $c-a+b-d \neq 0$, then $s_{0}-c=\frac{(d-c)(c-a)}{c-a+b-d}$ and $s_{0}-b=\frac{(d-b)(b-a)}{c-a+b-d}$, so we have four possible cases:

1. If $c-a+b-d>0$ (which implies $s_{0}>b, c$ ) and $x+n\left(s_{0}-x\right)<b$, we have

$$
I_{2}=\frac{(c-a+b-d)^{q}}{n^{q}(b-a)^{q}(d-c)^{q}} \int_{c}^{b}\left|(x-s)^{n-1}\left(x-s+n\left(s_{0}-x\right)\right)\right|^{q} d s .
$$

and

$$
\begin{aligned}
& \int_{c}^{b}\left|(x-s)^{n-1}\left(x-s+n\left(s_{0}-x\right)\right)\right|^{q} d s \\
= & \int_{c}^{x}(x-s)^{(n-1) q}\left(x-s+n\left(s_{0}-x\right)\right)^{q} d s \\
& +\int_{x}^{x+n\left(s_{0}-x\right)}(s-x)^{(n-1) q}\left(x-s+n\left(s_{0}-x\right)\right)^{q} d s \\
& +\int_{x+n\left(s_{0}-x\right)}^{b}(s-x)^{(n-1) q}\left(s-x+n\left(x-s_{0}\right)\right)^{q} d s \\
= & \left(s_{0}-x\right)^{q n+1} n^{q n+1}\left(\Psi \frac{x-c}{n\left(s_{0}-x\right)}((n-1) q+1, q+1)+B(q+1,(n-1) q+1)\right. \\
& +\Psi \frac{b-x}{n\left(s_{0}-x\right)}-1 \\
& (q+1,(n-1) q+1)) .
\end{aligned}
$$

2. If $c-a+b-d>0$ and $x+n\left(s_{0}-x\right)>b$, we have

$$
\begin{aligned}
& \int_{c}^{b}\left|(x-s)^{n-1}\left(x-s+n\left(s_{0}-x\right)\right)\right|^{q} d s \\
= & \int_{c}^{x}(x-s)^{(n-1) q}\left(x-s+n\left(s_{0}-x\right)\right)^{q} d s+\int_{x}^{b}(s-x)^{(n-1) q}\left(x-s+n\left(s_{0}-x\right)\right)^{q} d s \\
= & \left(s_{0}-x\right)^{q n+1} n^{q n+1}\left(\Psi_{\frac{x-c}{n\left(s_{0}-x\right)}}((n-1) q+1, q+1)\right. \\
& \left.+B(q+1,(n-1) q+1)-B_{1-\frac{b-x}{n\left(s_{0}-x\right)}}(q+1,(n-1) q+1)\right) .
\end{aligned}
$$


3. If $c-a+b-d<0$ (which implies $\left.s_{0}<b, c\right)$ and $x+n\left(s_{0}-x\right)>c$, we have

$$
I_{2}=\frac{(a-c+d-b)^{q}}{n^{q}(b-a)^{q}(d-c)^{q}} \int_{c}^{b}\left|(x-s)^{n-1}\left(s-x+n\left(x-s_{0}\right)\right)\right|^{q} d s
$$

and

$$
\begin{aligned}
& \int_{c}^{b}\left|(x-s)^{n-1}\left(x-s+n\left(s_{0}-x\right)\right)\right|^{q} d s \\
= & \int_{c}^{x+n\left(s_{0}-x\right)}(x-s)^{(n-1) q}\left(x-s+n\left(s_{0}-x\right)\right)^{q} d s \\
& +\int_{x+n\left(s_{0}-x\right)}^{x}(x-s)^{(n-1) q}\left(s-x+n\left(x-s_{0}\right)\right)^{q} d s \\
& +\int_{x}^{b}(s-x)^{(n-1) q}\left(s-x+n\left(x-s_{0}\right)\right)^{q} d s \\
= & \left(x-s_{0}\right)^{q n+1} n^{q n+1}\left(\Psi_{\frac{x-c}{n\left(x-s_{0}\right)}-1}(q+1,(n-1) q+1)\right. \\
& \left.+B(q+1,(n-1) q+1)+\Psi_{\frac{b-x}{n\left(x-s_{0}\right)}}((n-1) q+1, q+1)\right) .
\end{aligned}
$$

4. If $c-a+b-d<0$ and $x+n\left(s_{0}-x\right)<c$, we have

$$
\begin{aligned}
& \int_{c}^{b}\left|(x-s)^{n-1}\left(x-s+n\left(s_{0}-x\right)\right)\right|^{q} d s \\
= & \int_{c}^{x}(x-s)^{(n-1) q}\left(s-x+n\left(x-s_{0}\right)\right)^{q} d s+\int_{x}^{b}(s-x)^{(n-1) q}\left(s-x+n\left(x-s_{0}\right)\right)^{q} d s \\
= & \left(x-s_{0}\right)^{q n+1} n^{q n+1}\left(B(q+1,(n-1) q+1)-B_{1-\frac{x-c}{n\left(x-s_{0}\right)}}(q+1,(n-1) q+1)\right. \\
& \left.+\Psi_{\frac{b-x}{n\left(x-s_{0}\right)}}((n-1) q+1, q+1)\right) .
\end{aligned}
$$

Finally, by summing $I_{1}, I_{2}$, and $I_{3}$ the statement for $1<p \leq \infty$ follows. For $p=1$ we have

$$
\begin{aligned}
&\left\|K_{n}(x, s)\right\|_{\infty}= \sup _{s \in[a, d]}\left|K_{n}(x, s)\right| \\
&=\max \left\{\max _{s \in[a, c]}\left|-\frac{(x-s)^{n}}{n(b-a)}+\frac{x-a}{b-a}(x-s)^{n-1}\right|,\right. \\
& \max _{s \in[c, b]}\left|-\frac{(x-s)^{n}}{n(b-a)}+\frac{(x-s)^{n}}{n(d-c)}+\left(\frac{x-a}{b-a}-\frac{x-c}{d-c}\right)(x-s)^{n-1}\right|, \\
&\left.\max _{s \in[b, d]}\left|\frac{(x-s)^{n}}{n(d-c)}-\frac{x-d}{d-c}(x-s)^{n-1}\right|\right\} .
\end{aligned}
$$

By an elementary calculation we get

$$
\max _{s \in[a, c]}\left|-\frac{(x-s)^{n}}{n(b-a)}+\frac{x-a}{b-a}(x-s)^{n-1}\right|=\frac{(n-1)(x-a)^{n}}{n(b-a)},
$$




$$
\max _{s \in[b, d]}\left|\frac{(x-s)^{n}}{n(d-c)}-\frac{x-d}{d-c}(x-s)^{n-1}\right|=\frac{(n-1)(d-x)^{n}}{n(d-c)} .
$$

Again, for $\max _{s \in[c, b]}\left|-\frac{(x-s)^{n}}{n(b-a)}+\frac{(x-s)^{n}}{n(d-c)}+\left(\frac{x-a}{b-a}-\frac{x-c}{d-c}\right)(x-s)^{n-1}\right|$ we have five possible cases:

1. If $c-a+b-d>0$ (which implies $s_{0}>b, c$ ) and $x+n\left(s_{0}-x\right)<b$, we have

$$
\begin{aligned}
& \max _{s \in[c, b]}\left|-\frac{(x-s)^{n}}{n(b-a)}+\frac{(x-s)^{n}}{n(d-c)}+\left(\frac{x-a}{b-a}-\frac{x-c}{d-c}\right)(x-s)^{n-1}\right| \\
= & \frac{c-a+b-d}{n(b-a)(d-c)} \max _{s \in[c, b]}\left|(x-s)^{n-1}\left(x-s+n\left(s_{0}-x\right)\right)\right|
\end{aligned}
$$

and

$$
\begin{gathered}
\max _{s \in[c, x]}(x-s)^{n-1}\left(x-s+n\left(s_{0}-x\right)\right)=(x-c)^{n-1}\left(x-c+n\left(s_{0}-x\right)\right), \\
\max _{s \in\left[x, x+n\left(s_{0}-x\right)\right]}(s-x)^{n-1}\left(x-s+n\left(s_{0}-x\right)\right)=\left(s_{0}-x\right)^{n}(n-1)^{n-1}, \\
\max _{s \in\left[x+n\left(s_{0}-x\right), b\right]}(s-x)^{n-1}\left(s-x+n\left(x-s_{0}\right)\right)=(b-x)^{n-1}\left(b-x+n\left(x-s_{0}\right)\right) .
\end{gathered}
$$

2. If $c-a+b-d>0$ and $x+n\left(s_{0}-x\right)>b$, we have

$$
\begin{aligned}
& \max _{s \in[c, x]}(x-s)^{n-1}\left(x-s+n\left(s_{0}-x\right)\right)=(x-c)^{n-1}\left(x-c+n\left(s_{0}-x\right)\right), \\
& \max _{s \in[x, b]}(s-x)^{n-1}\left(x-s+n\left(s_{0}-x\right)\right)=(m-x)^{n-1}\left(x-m+n\left(s_{0}-x\right)\right)
\end{aligned}
$$

where $m=\min \left\{b, x+(n-1)\left(s_{0}-x\right)\right\}$.

3. If $c-a+b-d<0$ (which implies $\left.s_{0}<b, c\right)$ and $x+n\left(s_{0}-x\right)>c$, we have

$$
\begin{aligned}
& \max _{s \in[c, b]}\left|-\frac{(x-s)^{n}}{n(b-a)}+\frac{(x-s)^{n}}{n(d-c)}+\left(\frac{x-a}{b-a}-\frac{x-c}{d-c}\right)(x-s)^{n-1}\right| \\
= & \frac{a-c+d-b}{n(b-a)(d-c)} \max _{s \in[c, d]}\left|(x-s)^{n-1}\left(s-x+n\left(x-s_{0}\right)\right)\right|
\end{aligned}
$$

and

$$
\begin{gathered}
\max _{s \in\left[c, x+n\left(s_{0}-x\right)\right]}(x-s)^{n-1}\left(x-s+n\left(s_{0}-x\right)\right)=(x-c)^{n-1}\left(x-c+n\left(s_{0}-x\right)\right), \\
\max _{s \in\left[x+n\left(s_{0}-x\right), x\right]}(s-x)^{n-1}\left(x-s+n\left(s_{0}-x\right)\right)=\left(s_{0}-x\right)^{n}(n-1)^{n-1}, \\
\max _{s \in[x, b]}(s-x)^{n-1}\left(s-x+n\left(x-s_{0}\right)\right)=(b-x)^{n-1}\left(b-x+n\left(x-s_{0}\right)\right) .
\end{gathered}
$$

4. If $c-a+b-d<0$ and $x+n\left(s_{0}-x\right)<c$, we have

$$
\max _{s \in[c, x]}(x-s)^{n-1}\left(s-x+n\left(x-s_{0}\right)\right)=(x-M)^{n-1}\left(M-x+n\left(x-s_{0}\right)\right)
$$


where $M=\max \left\{c, x+(n-1)\left(s_{0}-x\right)\right\}$

$$
\max _{s \in[x, b]}(s-x)^{n-1}\left(s-x+n\left(x-s_{0}\right)\right)=(b-x)^{n-1}\left(b-x+n\left(x-s_{0}\right)\right) .
$$

5. If $c-a+b-d=0$

$$
\begin{aligned}
& \max _{s \in[c, b]}\left|-\frac{(x-s)^{n}}{n(b-a)}+\frac{(x-s)^{n}}{n(d-c)}+\left(\frac{x-a}{b-a}-\frac{x-c}{d-c}\right)(x-s)^{n-1}\right| \\
= & \frac{c-a}{b-a} \max _{s \in[c, b]}\left|(x-s)^{n-1}\right|=\max \left\{\frac{c-a}{b-a}(x-c)^{n-1}, \frac{c-a}{b-a}(b-x)^{n-1}\right\} .
\end{aligned}
$$

Thus, the proof is done.

Remark 5. If we put $b=c=x$ as a limit case, the inequalities from Corollary 3 reduce to

$$
\begin{aligned}
& \left|\frac{1}{x-a} \int_{a}^{x} f(t) d t-\frac{1}{d-x} \int_{x}^{d} f(t) d t+t_{n}^{[a, x]}(x)-t_{n}^{[x, d]}(x)\right| \\
\leq & \frac{1}{(n-1) !}\left[n^{(n-1) q+1}\left((x-a)^{(n-1) q+1}+(d-x)^{(n-1) q+1}\right)\right. \\
& \left.\cdot\left(B(q+1,(n-1) q+1)-B_{1-\frac{1}{n}}(q+1,(n-1) q+1)\right)\right]^{\frac{1}{q}}\left\|f^{(n)}\right\|_{p}
\end{aligned}
$$

for $1<p \leq \infty$ and for $p=1$

$$
\begin{aligned}
& \left|\frac{1}{x-a} \int_{a}^{x} f(t) d t-\frac{1}{d-x} \int_{x}^{d} f(t) d t+t_{n}^{[a, x]}(x)-t_{n}^{[x, d]}(x)\right| \\
\leq & \frac{1}{n !} \max \left\{(n-1)(x-a)^{n-1},(n-1)(d-x)^{n-1}\right\}\left\|f^{(n)}\right\|_{1} .
\end{aligned}
$$

Remark 6. If we suppose $b=d$ in both cases $[c, d] \subseteq[a, b]$ and $[a, b] \cap[c, d]=[c, b]$ the analogues results in Corollary 2 and Corollary 3 coincides.

\section{References}

[1] G. A. Anastassiou, Ostrowski type inequalities, Proc. Amer. Math. Soc. 123 (1995), 3775-3781.

[2] A. Aglić Aljinović and J. Pečarić, On some Ostrowski type inequalities via Montgomery identity and Taylor's formula, Tamkang Journal of Mathematics (accepted).

[3] N. S. Barnett, P. Cerone, S. S. Dragomir and A. M. Fink, Comparing two integral means for absolutely continuous mappings whose derivatives are in $L_{\infty}[a, b]$ and applications, Computers and Math.With Appl. 44 (2002), 241-251.

[4] M. Matić, J.Pečarić, Two-point Ostrowski inequality, Math. Inequal. Appl. 4(2001), 215221. 
[5] G. V. Milovanović, On some integral inequalities, Univ. Beograd, Publ. Elektrotehn. Fak. Ser. Mat. Fiz., No.498-541 (1975), 119-144.

[6] G. V. Milovanović, O nekim funkcionalnim nejednakostima, Univ. Beograd, Publ. Elektrotehn. Fak. Ser. Mat. Fiz., No.599 (1977), 1-59.

[7] D. S. Mitrinović, J. E. Pečarić and A. M. Fink, Inequalities for functions and their Integrals and Derivatives, Kluwer Academic Publishers, Dordrecht, 1994.

[8] J. Pečarić, On the Čebyšev inequality, Bul. Inst. Politehn. Timisoara 25 (39) (1980), 10-11.

Department of applied mathematics, Faculty of Electrical Engineering and Computing, University of Zagreb, Unska 3, 10000 Zagreb, Croatia.

E-mail: andrea@zpm.fer.hr

Faculty of textile technology, University of Zagreb, Pierottijeva 6, 10000 Zagreb, Croatia.

E-mail: pecaric@hazu.hr

Faculty of food technology and biotechnology, Mathematics department, University of Zagreb, Pierottijeva 6, 10000 Zagreb, Croatia.

E-mail: avukelic@pbf.hr 